\title{
Stability Analysis of Retarded Differential Inclusions
}

\author{
Jiafu Wang and Gui Zhang \\ Institute of Mathematics and Physics, Central South University of Forestry and Technology, Changsha, Hunan 410004, China \\ Correspondence should be addressed to Gui Zhang; gzhangmath@163.com
}

Received 29 December 2013; Accepted 9 March 2014; Published 1 April 2014

Academic Editor: Qiankun Song

Copyright (C) 2014 J. Wang and G. Zhang. This is an open access article distributed under the Creative Commons Attribution License, which permits unrestricted use, distribution, and reproduction in any medium, provided the original work is properly cited.

Retarded differential inclusions have drawn more and more attention, due to the development of feedback control systems with delays and dynamical systems determined by retarded differential equations with a discontinuous right-hand side. The purpose of this paper is to establish a result on the stability and asymptotical stability for retarded differential inclusions. Comparing with the previous results, the main result obtained in this paper allows Lyapunov functions to be nonsmooth. Moreover, to deal with the asymptotical stability, it is not required that Lyapunov functions should have an infinitesimal upper limit, but this condition is needed in most of the previous results. To demonstrate applicability, we use the main result in the analysis of asymptotical stability of a class of neural networks with discontinuous activations and delays.

\section{Introduction}

It has been known for ages that the future state of a system might depend not only on the present states but also on the past states [1-4]. In particular, in some problems it is meaningless not to have dependence on the past. Consequently, there is much concern about retarded differential equations:

$$
\dot{x}(t)=f\left(t, x_{t}\right),
$$

where "." represents the derivative, $x(t): \mathbb{R} \rightarrow \mathbb{R}^{n}$ is a vector-valued function, $x_{t} \in C\left([-r, 0], \mathbb{R}^{n}\right)$ is defined by $x_{t}(\theta)=x(t+\theta)$, and $f: \mathbb{R} \times C\left([-r, 0], \mathbb{R}^{n}\right) \rightarrow \mathbb{R}^{n}$ is a given function. To our knowledge, most results for (1) require the function $f$ to be continuous. However, in many applications the function $f$ may be discontinuous or even multivalued. For example, a lot of phenomena in biology are characterized by strongly localized coupling, that is, by interaction of an almost on-off nature; see [5-7]. Thus it is realistic to consider retarded differential equations with a discontinuous righthand side [8]. This gives rise to the study of systems described by retarded differential inclusions.

Another great impetus to study retarded differential inclusion comes from the development of control theory. A specific class of systems of retarded differential inclusion arising in technology consists of feedback control systems which can be described by equations of the form

$$
\dot{x}(t)=f\left(t, x_{t}, u\right),
$$

where $u=u\left(t, x_{t}\right)$ denotes controllers. Let $U$ be the set of allowable controllers and $G\left(t, x_{t}\right):=\left\{f\left(t, x_{t}, u\right)\right\}_{u \in U}$; then many of the qualitative properties of the control system (2) can be deduced from the corresponding qualitative properties of the system of retarded differential inclusions $\dot{x}(t) \in G\left(t, x_{t}\right)$. This approach has played an important role in investigating the absolute stability problem of regulator systems; see $[9,10]$.

In recent years, more and more attention has been drawn to the stability of the retarded differential inclusions; see [9-14]. In $[9,10]$, stability and asymptotical stability are investigated for retarded differential inclusions, and the Lyapunov functions are required to be differentiable. To study the asymptotical stability of retarded differential inclusions as well as ordinary differential inclusions, most of results in the literature are under the condition that the Lyapunov functions have an infinitesimal upper limit; see $[1,9,10,13]$, for example. In $[11,15]$, nonsmooth Lyapunov functions are successfully applied in discussing the stability of ordinary 
differential inclusions or ordinary differential equations with a discontinuous right-hand side. However, to the best of our knowledge, there is very little literature using nonsmooth Lyapunov functions to deal with the stability for retarded differential inclusions.

Based on these motivations, the objective of this paper is to make use of nonsmooth Lyapunov functions to study the stability of retarded differential inclusions. Dropping the condition that Lyapunov functions have an infinitesimal upper limit, we manage to obtain the asymptotical stability for retarded differential inclusions. Our method is based on the generalized Lyapunov approach introduced by $[15,16]$.

The outline of this paper is as follows. In Section 2, we give some preliminaries which are needed in this paper. Section 3 is devoted to investigate stability and asymptotical stability for retarded differential inclusions. In Section 4, an application of the main result obtained in Section 3 is given for analysis of stability of neural networks with discontinuous activations and delays.

\section{Preliminaries}

For $x=\left(x_{1}, x_{2}, \ldots, x_{n}\right)^{\mathrm{T}} \in \mathbb{R}^{n},\|x\|$ denotes a norm of $x$, where " $\mathrm{T}$ " is the transpose. Let $r \geq 0$ be a given real number and $C=C\left([-r, 0], \mathbb{R}^{n}\right)$ be the Banach space of continuous functions mapping the interval $[-r, 0]$ into $\mathbb{R}^{n}$. For an element $\phi \in C$, the norm of $\phi$ is defined by $|\phi|=$ $\sup _{-r \leq \theta \leq 0}|\phi(\theta)|$. If $b \geq 0$ and $x(t):[\sigma-r, \sigma+b] \rightarrow \mathbb{R}^{n}$ is continuous, then $x_{t} \in C$ is defined by $x_{t}(\theta)=x(t+\theta)$, $-r \leq \theta \leq 0$ for any $t \in[\sigma, \sigma+b]$. In the present paper, we consider the following retarded differential inclusion:

$$
\dot{x}(t) \in F\left(t, x_{t}\right)
$$

where $F: \mathbb{R} \times C \rightarrow \mathbb{R}^{n}$ is a set-valued map with nonempty convex compact values.

Definition 1. A function $x$ is said to be a solution of differential inclusion (3) on $[\sigma-r, \sigma+b)$ if there are $\sigma \in \mathbb{R}$ and $b>0$ such that $x(t)$ is continuous on $[\sigma-r, \sigma+b)$ and absolutely continuous on any compact subinterval of $[\sigma, \sigma+b)$ and satisfies (3) for almost all (a.a.) $t \in[\sigma, \sigma+b)$. For given $\sigma \in \mathbb{R}, \phi \in C, x(\sigma, \phi)(t)$ is said to be a solution of differential inclusion (3) with initial value $\phi$ at $\sigma$ if $x(\sigma, \phi)(t)$ is a solution of (3) on $[\sigma-r, \sigma+b)$ and $x_{\sigma}=\phi$.

It is remarked that, under the condition that the setvalued map $F$ in (3) is semicontinuous with nonempty convex compact values, a result on existence of solutions for (3) can be found in [17].

In order to investigate the stability of (3), we will use the generalized Lyapunov approach, so we borrow some basic notations and the chain rule for nonsmooth Lyapunov function from $[15,16]$.
A function $V: \mathbb{R}^{n} \rightarrow \mathbb{R}$, which is locally Lipschitz continuous at $x \in \mathbb{R}^{n}$, is said to be C-regular at $x$ if $V^{\prime}(x ; v)$ exists and $V^{\prime}(x ; v)=V^{\circ}(x ; v)$, where

$$
V^{\prime}(x ; v)=\lim _{\rho \rightarrow 0^{+}} \frac{V(x+\rho v)-V(x)}{\rho}
$$

is the directional derivative of $V$ at $x$ in the direction $v$ and

$$
V^{\circ}(x ; v)=\limsup _{\substack{y \rightarrow x \\ \rho \rightarrow 0^{+}}} \frac{V(y+\rho v)-V(y)}{\rho}
$$

is the generalized directional derivative of $V$ at $x$ in the direction $v$. The function $V$ is said to be C-regular in $\mathbb{R}^{n}$, if it is C-regular at any $x \in \mathbb{R}^{n}$. The generalized gradient $\partial V(x)$ of $V$ at $x \in \mathbb{R}^{n}$ is defined by

$$
\partial V(x)=\left\{\zeta \in \mathbb{R}^{n} \mid\langle\zeta, v\rangle \leq V^{\circ}(x ; v), \forall v \in \mathbb{R}^{n}\right\} .
$$

Lemma 2 (see [15]). If $V(x): \mathbb{R}^{n} \rightarrow \mathbb{R}$ is $C$-regular and $x(t):[\sigma,+\infty) \rightarrow \mathbb{R}^{n}$ is absolutely continuous on any compact subinterval of $[\sigma,+\infty)$ then for a.a. $t \in[\sigma,+\infty), x(t)$ and $V(x(t)):[\sigma,+\infty) \rightarrow \mathbb{R}$ are differentiable, and

$$
\dot{V}(x(t))=\langle\zeta(t), \dot{x}(t)\rangle, \quad \forall \zeta(t) \in \partial V(x(t)) .
$$

\section{Main Result}

In this section, we suppose $F(t, 0)=0$ and focus on the stability of the zero solution $x=0$ of the differential inclusion (3), since by a transformation the stability of any solution could be investigated in terms of the zero solution of the corresponding differential inclusion. The definition of stability of the solution $x=0$ can be given as in [1]. Let $\mathscr{B}(0, \delta)=\{\phi \in C|| \phi \mid<\delta\}$.

Definition 3. The solution $x=0$ of differential inclusion (3) is said to be stable if for any $\sigma \in \mathbb{R}, \varepsilon>0$, there is a $\delta=\delta(\varepsilon, \sigma)$ such that $\phi \in \mathscr{B}(0, \delta)$ implies $x_{t}(\sigma, \phi) \in \mathscr{B}(0, \varepsilon)$ for $t \geq \sigma$. The solution $x=0$ is said to be asymptotically stable if it is stable and there is a $b_{0}=b_{0}(\sigma)>0$ such that $\phi \in \mathscr{B}\left(0, b_{0}\right)$ implies $x(\sigma, \phi)(t) \rightarrow 0$ as $t \rightarrow \infty$. The solution $x=0$ is said to be globally asymptotically stable if it is stable and $x(\sigma, \phi)(t) \rightarrow$ 0 as $t \rightarrow \infty$ for any $\phi \in C$.

Let $V: \mathbb{R} \times \mathbb{R}^{n} \rightarrow \mathbb{R}^{n}$ be locally Lipschitz continuous and $x(\sigma, \phi)(t)$ is a solution of (3) with initial value $\phi$ at $\sigma$; we define

$$
\dot{V}(t, x)=\lim _{h \rightarrow 0} \frac{V(t+h, x(\sigma, \phi)(t+h))-V(t, x(\sigma, \phi)(t))}{h},
$$

where $\dot{V}(t, x)$ exists. The function $\dot{V}(t, x)$ is the derivative of $V(t, x)$ along the solution of (3).

Now we give the main result in this paper.

Theorem 4. Suppose that $F: \mathbb{R} \times C \rightarrow \mathbb{R}^{n}$ takes $\mathbb{R} \times$ (bounded sets of $C$ ) into bounded sets of $\mathbb{R}^{n}$, and $\varphi, \omega$ : $\mathbb{R}^{+} \rightarrow \mathbb{R}^{+}$are continuous nondecreasing functions, $\varphi(0)=0$ 
and $\varphi(s)>0$ for $s>0$. If there is a locally Lipschitz continuous function $V: \mathbb{R} \times \mathbb{R}^{n} \rightarrow \mathbb{R}$ such that

$$
V(t, x) \geq \varphi(\|x\|)
$$

and for any $\sigma \in \mathbb{R}$ and a.a. $t \in[\sigma,+\infty)$

$$
\dot{V}(t, x) \leq-\omega(\|x\|)
$$

then one has the following:

(i) the solution $x=0$ of the differential inclusion (3) is stable;

(ii) if $\omega(s)>0$ for $s>0$, the solution $x=0$ of the differential inclusion (3) is asymptotically stable;

(iii) if $\omega(s)>0$ for $s>0$ and $\varphi(s) \rightarrow \infty$ as $s \rightarrow \infty$, the solution $x=0$ of the differential inclusion (3) is globally asymptotically stable.

Proof. For any $\sigma \in \mathbb{R}$ and $\varepsilon>0$, there is a $\delta=\delta(\varepsilon$, $\sigma)$ such that $\phi \in \mathscr{B}(0, \delta)$ implies that $V(\sigma, \phi(0))<\varphi(\varepsilon / 2)$ from the continuity of the function $V$. Since $V(t, x)$ is locally Lipschitz continuous, we have that $V(t, x(\sigma, \phi)(t))$ is absolutely continuous on any compact subinterval of $[\sigma,+\infty)$ and

$$
\begin{aligned}
V(t, x(\sigma, \phi)(t))= & V\left(t_{0}, x(\sigma, \phi)\left(t_{0}\right)\right) \\
& +\int_{t_{0}}^{t} \dot{V}(s, x(\sigma, \phi)(s)) \mathrm{d} s, \quad \forall t_{0}, t \geq \sigma .
\end{aligned}
$$

It follows from (9) and (10) that

$$
\begin{aligned}
\varphi(\|x(\sigma, \phi)(t)\|) & \leq V(t, x(\sigma, \phi)(t)) \\
& \leq V(\sigma, \phi(0))<\varphi\left(\frac{\varepsilon}{2}\right), \quad t \geq \sigma,
\end{aligned}
$$

which means that

$$
\|x(\sigma, \phi)(t)\| \leq \frac{\varepsilon}{2}<\varepsilon, \quad t \geq \sigma,
$$

and thus $x_{t}(\sigma, \phi) \in \mathscr{B}(0, \varepsilon)$ for $t \geq \sigma$. This proves the stability.

In order to prove the assertion (ii), we need only to show that there is a $b_{0}=b_{0}(\sigma)>0$ such that

$$
\lim _{t \rightarrow \infty} x(\sigma, \phi)(t)=0
$$

for any $\phi \in \mathscr{B}\left(0, b_{0}\right)$. Using the approach of contradiction, assume for any $b_{0}>0$ there is $\phi \in \mathscr{B}\left(0, b_{0}\right)$ such that (14) fails to hold; then there exist $\varepsilon_{0}>0$ and a sequence $\left\{t_{k}\right\}$ such that $t_{k} \rightarrow \infty$ as $k \rightarrow \infty$ and

$$
\left\|x(\sigma, \phi)\left(t_{k}\right)\right\| \geq \varepsilon_{0} .
$$

Note that, as long as $b_{0}$ is small enough, there is $M>0$ such that $V(\sigma, \phi(0))<\varphi(M)$. It follows from (9) and (10) that

$$
\begin{aligned}
\varphi(\|x(\sigma, \phi)(t)\|) & \leq V(t, x(\sigma, \phi)(t)) \leq V(\sigma, \phi(0)) \\
& <\varphi(M),
\end{aligned}
$$

and thus

$$
\|x(\sigma, \phi)(t)\| \leq M
$$

which means $x(\sigma, \phi)(t)$ is bounded. Notice that the map $F$ : $\mathbb{R} \times C \rightarrow \mathbb{R}^{n}$ takes $\mathbb{R} \times$ (bounded sets of $C$ ) into bounded sets of $\mathbb{R}^{n}$, there is $L>0$ such that

$$
\|\dot{x}(\sigma, \phi)(t)\|<L, \quad t \geq \sigma .
$$

Take $l=\varepsilon_{0} / 2 L$; then for $t \in\left[t_{k}-l, t_{k}+l\right]$

$$
\|x(\sigma, \phi)(t)\| \geq\left\|x(\sigma, \phi)\left(t_{k}\right)\right\|-\left\|\int_{t_{k}}^{t} \dot{x}(\sigma, \phi)(s) \mathrm{d} s\right\|
$$

and hence from (15) and (18)

$$
\begin{array}{r}
\|x(\sigma, \phi)(t)\| \geq \varepsilon_{0}-L\left|t-t_{k}\right| \geq \frac{\varepsilon_{0}}{2}, \\
t \in\left[t_{k}-l, t_{k}+l\right] .
\end{array}
$$

Therefore, by (10) we have for a.a. $t \in\left[t_{k}-l, t_{k}+l\right]$

$$
\dot{V}(t, x(\sigma, \phi)(t)) \leq-\omega\left(\frac{\varepsilon_{0}}{2}\right) \text {. }
$$

By taking a subsequence of $\left\{t_{k}\right\}$, if necessary, we can assume that $t_{1}-l>\sigma$ and the intervals $\left[t_{k}-l, t_{k}+l\right]$ do not overlap, and (10) implies that

$$
\begin{aligned}
& V\left(t_{k}, x(\sigma, \phi)\left(t_{k}\right)\right) \\
& \quad=V(\sigma, \phi(0))+\int_{\sigma}^{t_{k}} \dot{V}(s, x(\sigma, \phi)(s)) \mathrm{d} s \\
& \quad \leq V(\sigma, \phi(0))-\sum_{i=1}^{k} \int_{t_{i}-l}^{t_{i}} \omega\left(\frac{\varepsilon_{0}}{2}\right) \mathrm{d} s \\
& \quad=V(\sigma, \phi(0))-k l \omega\left(\frac{\varepsilon_{0}}{2}\right) .
\end{aligned}
$$

If $k>V(\sigma, \phi(0)) / l \omega\left(\varepsilon_{0} / 2\right)$, then

$$
V\left(t_{k}, x(\sigma, \phi)\left(t_{k}\right)\right)<0,
$$

which is a contradiction. This proves the asymptotical stability.

It is clear that if $\varphi(s) \rightarrow \infty$ as $s \rightarrow \infty$, then (14) is true for all $\phi \in C$. Therefore, the solution $x=0$ is globally asymptotically stable.

Take $\omega(s) \equiv 0$; then $\omega(s)$ is a continuous nondecreasing function. Thus we have the following remark.

Remark 5. The conclusion (i) of Theorem 4 also holds, if the condition (10) is replaced by the following simple condition:

$$
\dot{V}(t, x) \leq 0
$$


Remark 6. Comparing with previous stability results in $[1,9$, $10,15]$, the advantages of Theorem 4 are as follows.

(i) Theorem 4 can be used to deal with the stability for retarded differential inclusions or retarded differential equations with a discontinuous right-hand side (see Section 4).

(ii) Theorem 4 allows us to use nonsmooth Lyapunov function to discuss the stability.

(iii) To investigate the asymptotical stability, Theorem 4 drops the condition that the Lyapunov function should have an infinitesimal upper limit; that is, there exists a function $\varphi_{2}: \mathbb{R}^{+} \rightarrow \mathbb{R}^{+}$such that $\varphi_{2}(0)=0$, $\varphi_{2}(s)>0$ for $s>0$, and

$$
V(t, x) \leq \varphi_{2}(\|x\|) .
$$

\section{Application}

In this section, an application of the main result obtained in Section 3 is given for analysis of stability of retarded neural networks with discontinuous activations.

Consider the retarded neural network which is described by the following differential equation:

$$
\begin{aligned}
& \dot{x}_{1}(t)=-x_{1}(t)-a g\left(x_{1}(t)\right)+b g\left(x_{2}(t-1)\right), \\
& \dot{x}_{2}(t)=-x_{2}(t)-a g\left(x_{2}(t)\right)+b g\left(x_{1}(t-1)\right),
\end{aligned}
$$

where $x_{1}$ and $x_{2}$ are neuron states, $a$ and $b$ are constants representing the neuron interconnection coefficients, and $g(s): \mathbb{R} \rightarrow \mathbb{R}$ is the neuron input-output activation, which is defined by

$$
g(s)=\operatorname{sgn}(s)= \begin{cases}1, & s>0 \\ 0, & s=0 \\ -1, & s<0\end{cases}
$$

Following [18, 19], $x(t)=\left(x_{1}(t), x_{2}(t)\right)^{\mathrm{T}}$ is a solution of the system $(26)$ on $[\sigma-1,+\infty)$ with initial value $\phi$ at $\sigma$, if $x$ is continuous on $[\sigma-1,+\infty)$ and absolutely continuous on any compact subinterval of $[\sigma,+\infty)$, and there exists a measurable function $\gamma(t)=\left(\gamma_{1}(t), \gamma_{2}(t)\right)^{\mathrm{T}}:[\sigma-1,+\infty) \rightarrow$ $\mathbb{R}^{2}$ such that $\gamma_{i}(t) \in K\left[g\left(x_{i}(t)\right)\right], i=1,2$, for a.a. $t \in[\sigma-$ $1,+\infty)$ and

$$
\begin{aligned}
& \dot{x}_{1}(t)=-x_{1}(t)-a \gamma_{1}(t)+b \gamma_{2}(t-1), \\
& \dot{x}_{2}(t)=-x_{2}(t)-a \gamma_{2}(t)+b \gamma_{1}(t-1),
\end{aligned}
$$

where

$$
K[g(s)]= \begin{cases}\{1\}, & s>0 \\ {[-1,1],} & s=0 \\ \{-1\}, & s<0\end{cases}
$$

and $\gamma(t)$ is called an output associated with the solution $x(t)$.

It is clear that if $x(t)$ is a solution of the system (26), then it is a solution of the following retarded differential inclusion:

$$
\dot{x}(t) \in F\left(t, x_{t}\right) \text {, }
$$

where

$$
F\left(t, x_{t}\right)=\left(\begin{array}{l}
-x_{1}(t)-a K\left[g\left(x_{1}(t)\right)\right]+b K\left[g\left(x_{2}(t-1)\right)\right] \\
-x_{2}(t)-a K\left[g\left(x_{2}(t)\right)\right]+b K\left[g\left(x_{1}(t-1)\right)\right]
\end{array}\right) .
$$

It is evident that $x=(0,0)^{\mathrm{T}}$ is a solution of the system (26). Next we will use Theorem 4 to show that the solution $x=(0,0)^{\mathrm{T}}$ is globally asymptotically stable if $a \geq|b|$.

Let $x(t)=\left(x_{1}(t), x_{2}(t)\right)^{\mathrm{T}}$ be a solution of the system (26) on $[\sigma-1,+\infty)$ with initial value $\phi$ at $\sigma$ and let the output associated with $x(t)$ be $\gamma(t)=\left(\gamma_{1}(t), \gamma_{2}(t)\right)^{\mathrm{T}}$. Consider the function

$$
V\left(t, x_{1}, x_{2}\right)=V_{1}\left(x_{1}, x_{2}\right)+|b| V_{2}(t)
$$

where

$$
\begin{gathered}
V_{1}\left(x_{1}, x_{2}\right)=\|x\|=\left|x_{1}\right|+\left|x_{2}\right|, \\
V_{2}(t)=\int_{t-1}^{t}\left(\left|\gamma_{1}(s)\right|+\left|\gamma_{2}(s)\right|\right) \mathrm{d} s ;
\end{gathered}
$$

then $V\left(t, x_{1}, x_{2}\right) \geq\|x\|, V_{1}\left(x_{1}, x_{2}\right)$ is regular, and the generalized gradient of $V_{1}$ at $\left(x_{1}, x_{2}\right)$ is

$$
\partial V_{1}\left(x_{1}, x_{2}\right)=\left\{\left(\eta_{1}, \eta_{2}\right)^{\mathrm{T}} \mid \eta_{i} \in K\left[g\left(x_{i}\right)\right], i=1,2\right\} .
$$

It follows from Lemma 2 that $V_{1}\left(x_{1}(t), x_{2}(t)\right)$ is differentiable and

$$
\begin{array}{r}
\dot{V}_{1}\left(x_{1}, x_{2}\right)=\left\langle\zeta(t),\left(\dot{x}_{1}(t), \dot{x}_{2}(t)\right)^{\mathrm{T}}\right\rangle, \\
\forall \zeta(t) \in \partial V_{1}\left(x_{1}(t), x_{2}(t)\right)
\end{array}
$$

for a.a. $t \in[\sigma,+\infty)$. Define

$$
v_{i}(t)= \begin{cases}0, & x_{i}(t)=\gamma_{i}(t)=0, \\ \operatorname{sgn}\left(\gamma_{i}(t)\right), & x_{i}(t)=0, \gamma_{i}(t) \neq 0, \\ \operatorname{sgn}\left(x_{i}(t)\right), & x_{i}(t) \neq 0,\end{cases}
$$

$i=1,2$; then $v_{i} x_{i}=\left|x_{i}\right|, v_{i} \gamma_{i}=\left|\gamma_{i}\right|$, and $\zeta(t)=\left(v_{1}(t), v_{2}(t)\right)^{\mathrm{T}} \epsilon$ $\partial V_{1}\left(x_{1}(t), x_{2}(t)\right)$. Note that $a \geq|b|$; we have

$$
\begin{aligned}
\dot{V}( & \left.t, x_{1}, x_{2}\right) \\
= & \dot{V}_{1}\left(x_{1}, x_{2}\right)+|b| \dot{V}_{2}(t) \\
= & v_{1}(t) \dot{x}_{1}(t)+v_{2}(t) \dot{x}_{2}(t) \\
& +|b|\left(\left|\gamma_{1}(t)\right|+\left|\gamma_{2}(t)\right|-\left|\gamma_{1}(t-1)\right|-\left|\gamma_{2}(t-1)\right|\right) \\
= & -\left|x_{1}(t)\right|-\left|x_{2}(t)\right|-(a-|b|)\left|\gamma_{1}(t)\right| \\
& -(a-|b|)\left|\gamma_{2}(t)\right|+v_{1}(t) b \gamma_{1}(t-1) \\
& +v_{2}(t) b \gamma_{2}(t-1)-|b|\left|\gamma_{1}(t-1)\right|-|b|\left|\gamma_{2}(t-1)\right| \\
\leq & -\left(\left|x_{1}(t)\right|+\left|x_{2}(t)\right|\right)=-\omega(\|x(t)\|),
\end{aligned}
$$

where $\omega(s)=s$; thus it follows from Theorem 4 that the solution $x=(0,0)^{\mathrm{T}}$ is globally asymptotically stable; see Figure 1 . 


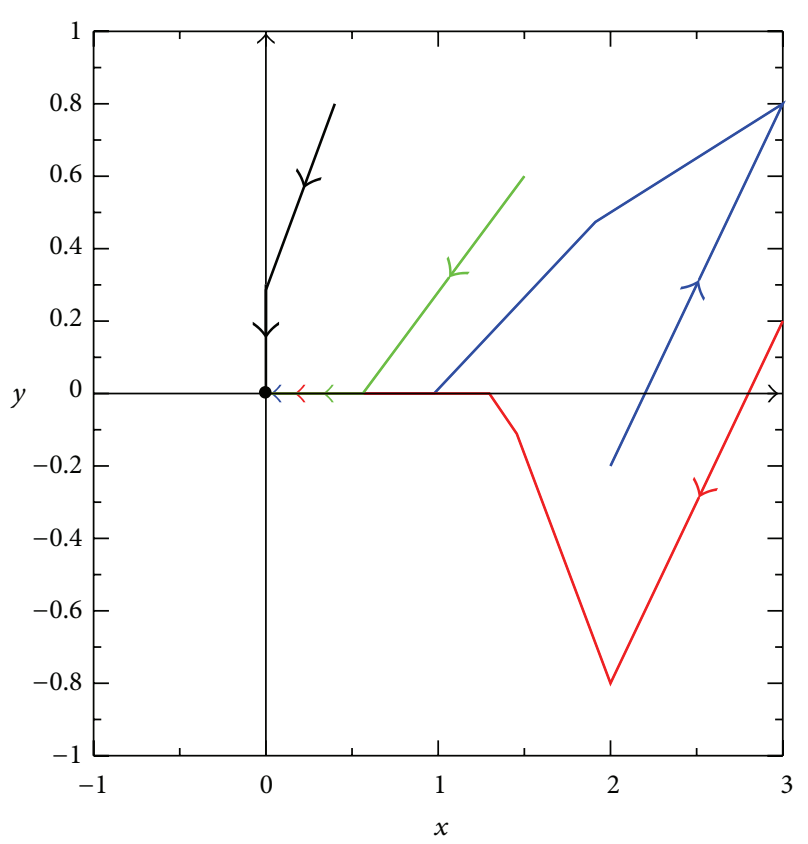

FIGURE 1: Dynamical behavior of (26) where initial conditions are chosen as $(\theta+3, \theta+0.8),(-\theta+2,-\theta-0.8),(0.4,0.8)$, and $(1.5,0.6)$ for $\theta \in[-1,0]$, and the parameters are fixed as $a=2$ and $b=1$.

\section{Conflict of Interests}

The authors declare that there is no conflict of interests regarding the publication of this paper.

\section{Acknowledgments}

This work is supported by the National Natural Science Foundation of China (Grant nos. 11301551 and 11226151) and by Hunan Provincial Natural Science Foundation of China (13JJ4088).

\section{References}

[1] J. K. Hale and S. M. Lunel, Introduction to FunctionalDifferential Equations, Springer, New York, NY, USA, 1993.

[2] U. Foryś, "Global stability for a class of delay differential equations," Applied Mathematics Letters, vol. 17, no. 5, pp. 581$584,2004$.

[3] J. Wang and L. Huang, "Almost periodicity for a class of delayed Cohen-Grossberg neural networks with discontinuous activations," Chaos, Solitons \& Fractals, vol. 45, no. 9-10, pp. 1157-1170, 2012.

[4] J. Qi and Y. Sun, "Global exponential stability of certain switched systems with time-varying delays," Applied Mathematics Letters, vol. 26, no. 7, pp. 760-765, 2013.

[5] H. de Jong, J. Geiselmann, G. Batt, C. Hernandez, and M. Page, "Qualitative simulation of the initiation of sporulation in Bacillus subtilis," Bulletin of Mathematical Biology, vol. 66, no. 2, pp. 261-299, 2004.

[6] T. Gedeon, "Global dynamics of neural nets with infinite gain," Physica D. Nonlinear Phenomena, vol. 146, no. 1-4, pp. 200-212, 2000.
[7] L. Glass, "Combinatorial and topological methods in nonlinear chemical kinetics," The Journal of Chemical Physics, vol. 63, no. 4, pp. 1325-1335, 1975.

[8] A. F. Filippov, Differential Equations with Discontinuous Righthand Sides, Kluwer Academic, Boston, Mass, USA, 1988.

[9] K. Wang and A. N. Michel, "Stability analysis of differential inclusions in Banach space with applications to nonlinear systems with time delays," IEEE Transactions on Circuits and Systems. I. Fundamental Theory and Applications, vol. 43, no. 8, pp. 617-626, 1996.

[10] A. Surkov, "On the stability of functional-differential inclusions with the use of invariantly differentiable lyapunov functionals," Differential Equations, vol. 43, no. 8, pp. 1079-1087, 2007.

[11] A. Bacciotti and F. Ceragioli, "Stability and stabilization of discontinuous systems and nonsmooth Lyapunov functions," ESAIM. Control, Optimisation and Calculus of Variations. European Series in Applied and Industrial Mathematics, vol. 4, pp. 361-376, 1999.

[12] A. N. Michel and Y. Sun, "Stability of discontinuous Cauchy problems in Banach space," Nonlinear Analysis. Theory, Methods \& Applications. Series A: Theory and Methods, vol. 65, no. 9, pp. 1805-1832, 2006.

[13] L. Hou and A. N. Michel, "Unifying theory for stability of continuous, discontinuous, and discrete-time dynamical systems," Nonlinear Analysis. Hybrid Systems, vol. 1, no. 2, pp. 154-172, 2007.

[14] Y.-J. Sun, "Stability criteria for a class of differential inclusion systems with discrete and distributed time delays," Chaos, Solitons and Fractals, vol. 39, no. 5, pp. 2386-2391, 2009.

[15] M. Forti, M. Grazzini, P. Nistri, and L. Pancioni, "Generalized Lyapunov approach for convergence of neural networks with discontinuous or non-Lipschitz activations," Physica D. Nonlinear Phenomena, vol. 214, no. 1, pp. 88-99, 2006.

[16] F. H. Clarke, Optimization and Nonsmooth Analysis, John Wiley \& Sons, New York, NY, USA, 1983.

[17] G. Haddad, "Monotone viable trajectories for functionaldifferential inclusions," Journal of Differential Equations, vol. 42, no. 1, pp. 1-24, 1981.

[18] M. Forti, P. Nistri, and D. Papini, "Global exponential stability and global convergence in finite time of delayed neural networks with infinite gain," IEEE Transactions on Neural Networks, vol. 16, no. 6, pp. 1449-1463, 2005.

[19] W. Lu and T. Chen, "Dynamical behaviors of delayed neural network systems with discontinuous activation functions," Neural Computation, vol. 18, no. 3, pp. 683-708, 2006. 


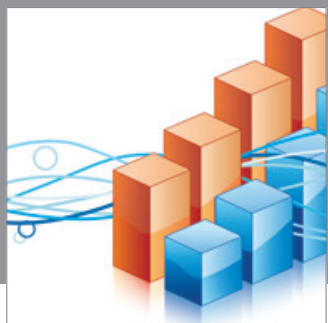

Advances in

Operations Research

mansans

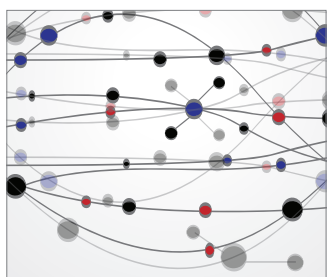

The Scientific World Journal
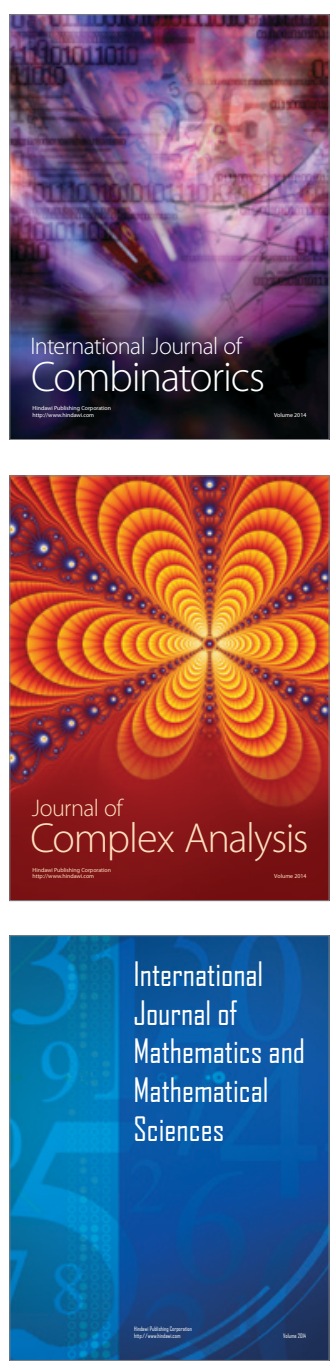
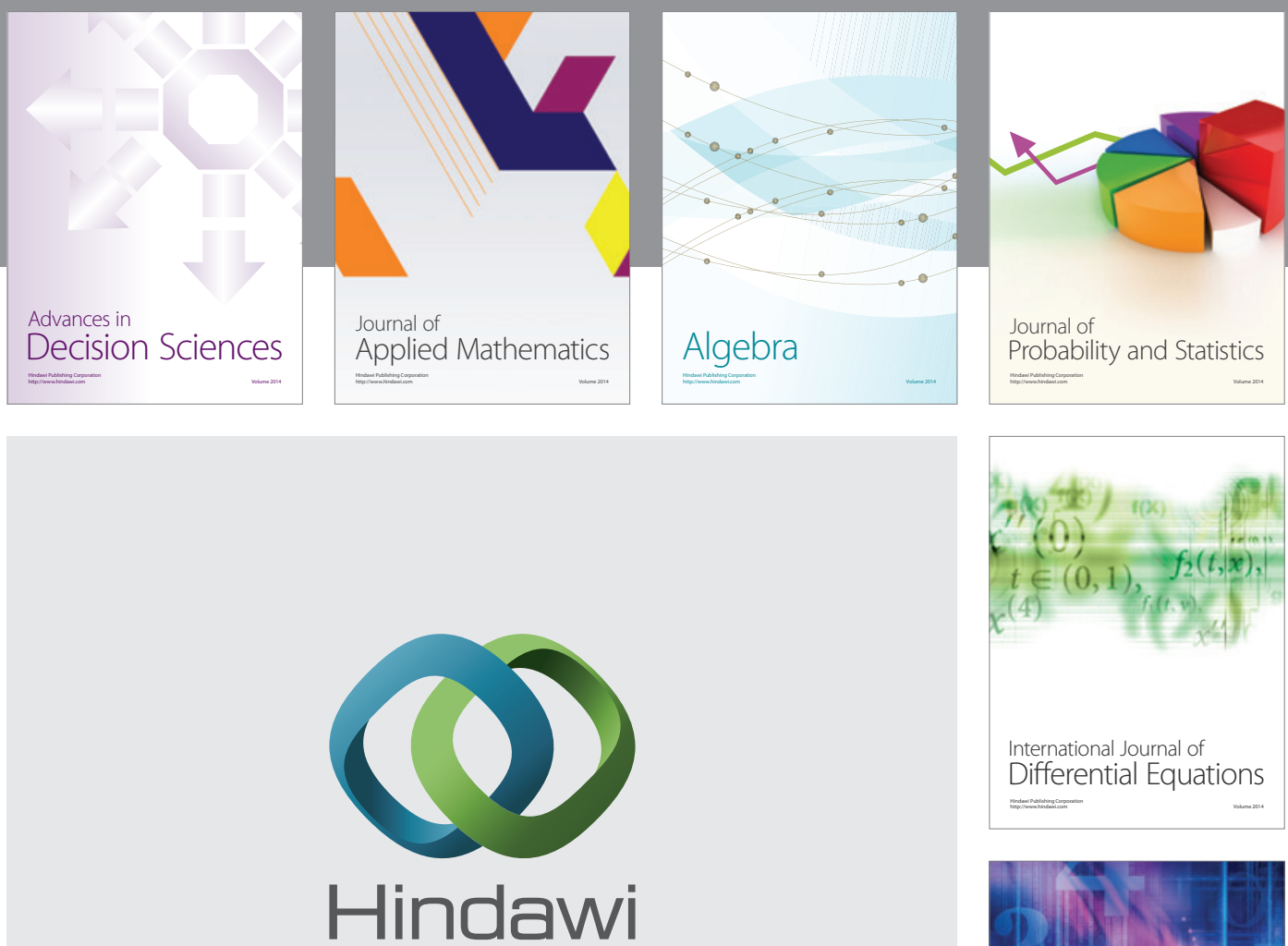

Submit your manuscripts at http://www.hindawi.com
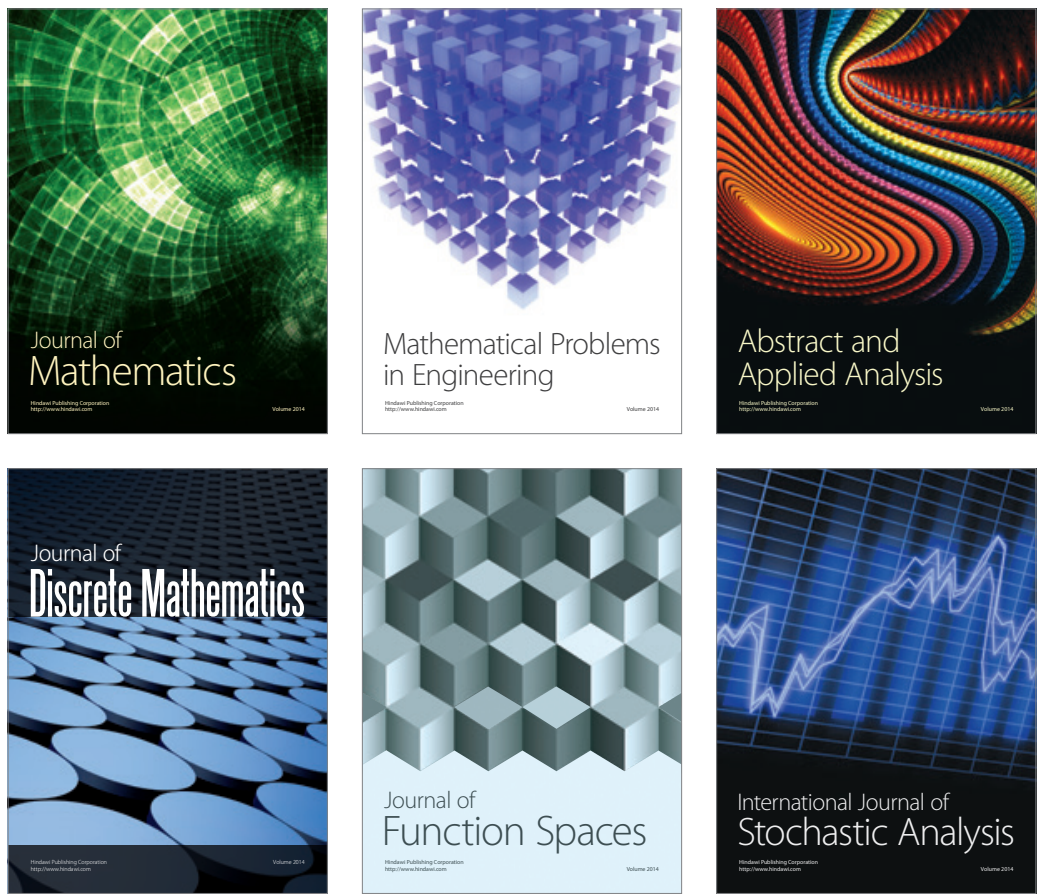

Journal of

Function Spaces

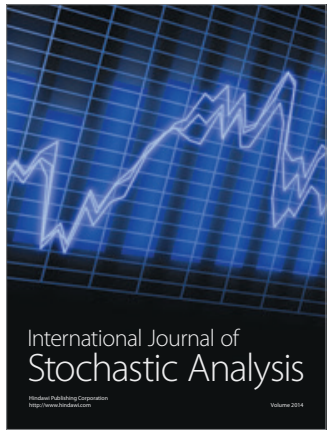

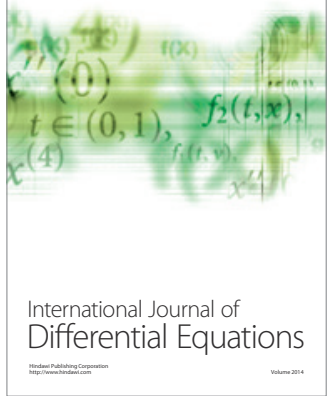
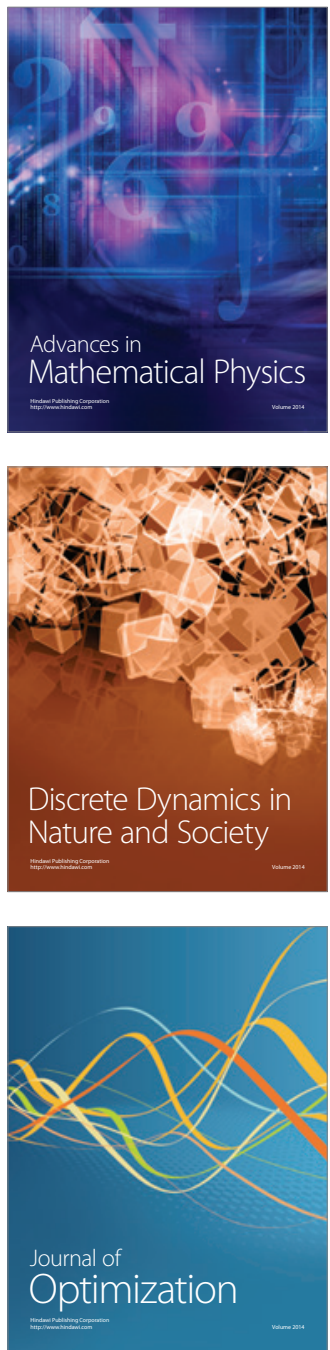